\title{
Isolated intersection multiplicity and regular separation of analytic sets
}

\author{
by Piotr Tworzewski (Kraków)
}

\begin{abstract}
An isolated point of intersection of two analytic sets is considered. We give a sharp estimate of their regular separation exponent in terms of intersection multiplicity and local degrees.
\end{abstract}

1. Separation. Let $M$ be an $m$-dimensional normed complex vector space. Following ([4], IV.7) we say that a pair of closed sets $X, Y$ in an open subset $G$ of $M$ satisfies the condition (S) at a point $a \in G$ if either $a \notin X \cap Y$, or $a \in X \cap Y$ and

$$
\varrho(z, X)+\varrho(z, Y) \geq c \varrho(z, X \cap Y)^{p}
$$

for $z$ in a neighbourhood of $a$, for some $c, p>0(\varrho(\cdot, Z)$ denotes the distance function to the set $Z \subset M)$.

In the sequel we will consider only isolated points of the intersection of $X$ and $Y$.

We say that $X$ and $Y$ are $p$-separated at $a \in G$ if $a$ is an isolated point of $X \cap Y$ and the pair $X, Y$ satisfies the condition (S) at $a$, with exponent $p$ and some constant $c>0$.

As a simple consequence of properties of (S) (see [4], IV.7.1) we get the following lemma.

Lemma 1.1. Let $H_{1} \subset G$ and $H_{2}$ be open subsets of normed, finitedimensional complex vector spaces and let $f: H_{1} \rightarrow H_{2}$ be a biholomorphism. Then closed subsets $X$ and $Y$ of $G$ are p-separated at a point $a \in H_{1}$ if and only if $f\left(X \cap H_{1}\right)$ and $f\left(Y \cap H_{1}\right)$ are $p$-separated at $f(a)$.

1991 Mathematics Subject Classification: 32B10, 14C17.

Key words and phrases: improper isolated intersection, multiplicity, exponent of regular separation.

Supported by KBN grant 210779101 . 
By the above lemma our condition can be carried over - in a classical manner - to the case of manifolds. (In this paper all manifolds are assumed to be second-countable.)

Namely, we say that closed subsets $X, Y$ of an $m$-dimensional complex manifold $M$ are $p$-separated at $a \in M$ if for some (and hence for every) chart $\varphi: \Omega \rightarrow G \subset \mathbb{C}^{m}$ such that $a \in \Omega$, the sets $\varphi(X \cap \Omega), \varphi(Y \cap \Omega)$, closed in $G$, are $p$-separated at $\varphi(a)$.

It is clear that if $X$ and $Y$ are $p$-separated at $a \in M$ and $X \cap Y=\{a\}$, then the pair $X, Y$ satisfies the "condition of regular separation" (see [4], IV.7.1).

Now, suppose that $X$ and $Y$ are analytic subsets of $M$ and $a \in M$ is an isolated point of $X \cap Y$. The principal topic of our research is a detailed study of the set

$$
P=\{p>0: X \text { and } Y \text { are } p \text {-separated at } a\},
$$

and of the best exponent

$$
p_{0}=p_{0}(X, Y ; a)=\inf P .
$$

If $\operatorname{dim} M=m \geq 1$, then a standard calculation yields $p_{0} \geq 1$. Obviously, $p_{0}=0$ for $m=0$.

LEMMA 1.2. Let $M$ be an open subset of a normed, finite-dimensional complex vector space. Suppose that $a$ is an accumulation point of $X$. Then $X$ and $Y$ are $p$-separated at $a$ if and only if there exists a neighbourhood $U$ of $a$ and $c>0$ such that

$$
\varrho(x, Y) \geq c|x-a|^{p} \quad \text { for } x \in X \cap U .
$$

P r o o f. It suffices to show that the above condition implies that $X$ and $Y$ are $p$-separated at $a$. Without loss of generality we can assume that $c \in(0,1)$ and $U$ is contained in the ball $B(a, 1)$. Since $a$ is an accumulation point of $X$, we see that $p \geq 1$.

Fix $r>0$ such that $B(a, 2 r) \subset U$. If $z \in B(a, r)$ then there exist $x \in X \cap$ $B(a, 2 r)$ and $y \in Y \cap B(a, 2 r)$ such that $\varrho(z, X)=|z-x|$ and $\varrho(z, Y)=|z-y|$. An easy computation shows that

$$
l=\varrho(z, X)+\varrho(z, Y) \geq|x-y| \geq \varrho(x, Y) \geq c|x-a|^{p} .
$$

Moreover,

$$
l \geq \varrho(z, X)=|z-x| \geq c|z-x|^{p} .
$$

Combining these inequalities we deduce that

$$
l \geq \frac{c}{2}\left(|x-a|^{p}+|z-x|^{p}\right) \geq \frac{c}{2^{p}}|z-a|^{p} \quad \text { for } z \in B(a, r),
$$

and the proof is complete.

We now state a result which we shall frequently use. 
Lemma 1.3. Let $M$ be a complex manifold. If $a \in M$ and $p>0$ then the following conditions are equivalent:

(1) $X$ and $Y$ are $p$-separated at $a$,

(2) $X \times Y$ and $\Delta_{M}$ are $p$-separated at $(a, a)$, where $\Delta_{M}=\left\{(x, x) \in M^{2}: x \in M\right\}$ is the diagonal in $M^{2}$.

Proof. Without loss of generality we can assume that $M$ is an open subset of a normed complex vector space $N$ with $\operatorname{dim} N \geq 1$.

Consider $N^{2}$ with the norm $|(x, y)|=|x|+|y|$. Observe that, for $z \in M$,

$$
\varrho((z, z), X \times Y)=\varrho(z, X)+\varrho(z, Y), \quad|(z, z)-(a, a)|=2|z-a| .
$$

Lemma 1.2 now shows that condition (2) is satisfied if and only if

$$
\varrho(z, X)+\varrho(z, Y) \geq c|z-a|^{p},
$$

in a neighbourhood of $a$, for some $c>0$. This completes the proof.

2. Multiplicity of isolated intersection. For the convenience of the reader we repeat, from [1], basic definitions and facts on isolated intersections of analytic sets.

Let $Z$ be a pure $k$-dimensional locally analytic subset of a complex manifold $M$ of dimension $m$. Let $N$ be a submanifold of $M$ of dimension $n$ such that $N$ intersects $Z$ at an isolated point $a \in M$. We denote by $\mathcal{F}_{a}(Z, N)$ the set of all locally analytic subsets $V$ of $M$ satisfying:

(1) $V$ has pure dimension $m-k$,

(2) $N_{a} \subset V_{a}$,

(3) $a$ is an isolated point of $V \cap Z$,

where $N_{a}, V_{a}$ denote the germs of $N$ and $V$ at $a$.

Observe that for $V \in \mathcal{F}_{a}(Z, N)$ the intersection of $Z$ and $V$ is proper at $a$ and we can consider the classical intersection multiplicity $i(Z \cdot V ; a)$ in the sense of Draper [2] (cf. [9]). We define

$$
\begin{aligned}
\widetilde{\mathfrak{i}}(Z \cdot N ; a) & =\min \left\{\mathfrak{i}(Z \cdot V ; a): V \in \mathcal{F}_{a}(Z, N)\right\}, \\
\mathcal{P}_{a}(Z, N) & =\left\{V \in \mathcal{F}_{a}(Z, N): \mathfrak{i}(Z \cdot V ; a)=\widetilde{\mathfrak{i}}(Z \cdot N ; a)\right\} .
\end{aligned}
$$

Note that ([1], Th. 4.4) gives the full characterization of the family $\mathcal{P}_{a}(Z, N)$.

Having disposed of this preliminary step we can now turn to the general case. Let $X, Y$ be pure dimensional locally analytic subsets of a complex manifold $M$ such that $a$ is an isolated point of $X \cap Y$. The positive integer

$$
\mathfrak{i}(X \cdot Y ; a)=\tilde{\mathfrak{i}}\left((X \times Y) \cdot \Delta_{M} ;(a, a)\right)
$$

is defined to be the multiplicity of intersection of $X$ and $Y$ at $a$.

If $Y$ is a submanifold the definition of $i(X \cdot Y ; a)$ presented above coincides with that of $\widetilde{i}(X \cdot Y ; a)$ introduced earlier. 
Finally, observe that in the case $Y=\{a\}$ we get

$$
i(X \cdot Y ; a)=\widetilde{i}(X \cdot Y ; a)=\operatorname{deg}_{a} X,
$$

where $\operatorname{deg}_{a} X$ is the classical degree (the Lelong number) of $X$ at $a$ (see e.g. $[1],[2])$.

3. Main results. In this part we apply the "diagonal construction" to separation of analytic sets. Let us begin with the following theorem motivated by [7].

TheOrem 3.1. Let $Z$ be a pure dimensional analytic subset and let $N$ be a closed submanifold of a complex manifold $M$ of dimension $m \geq 1$. Suppose that $a \in M$ is an isolated point of $Z \cap N$ and set

$$
P=\{p>0: Z \text { and } N \text { are } p \text {-separated at } a\} \text {. }
$$

Then

1) $p_{0}=\inf P \in P \cap \mathbb{Q}$

2) $1 \leq p_{0} \leq i(Z \cdot N ; a)-\operatorname{deg}_{a} Z+1$.

Proof. Let $V \in \mathcal{P}_{a}(Z ; N)$ (see Section 2$)$. We know that $i(Z \cdot N ; a)=$ $i(V \cdot N ; a)$, and ([1], Th. 4.4) implies that $V_{a}$ is a germ of a manifold. Suppose that $\operatorname{dim} Z=k, \operatorname{dim} N=n$.

We can assume, by using Lemma 1.1 if necessary, that:

- $M=B \times D \times \mathbb{C}^{n}$, where $B$ and $D$ are the unit balls in $\mathbb{C}^{k}, \mathbb{C}^{m-n-k}$ respectively,

- $N=\{0\} \times \mathbb{C}^{n}, 0 \in \mathbb{C}^{m-n}$,

- $V=\{0\} \times D \times \mathbb{C}^{n}, 0 \in \mathbb{C}^{k}$,

- $Z \cap V=\{0\}$,

- $\pi \mid Z: Z \rightarrow B \times D$ is proper, where $\pi: M \rightarrow B \times D$ is the natural projection.

In this situation, by ([1], Th. 4.4, Lemma 2.4), we obtain $C_{0}(\pi(Z)) \cap$ $(\{0\} \times D)=\{0\}$, where $C_{0}(\pi(Z))$ is the tangent cone of the set $\pi(Z)$ at $0 \in \mathbb{C}^{m-n}$. An easy computation and ([7], Th. (1.2)) show that there exists an open neighbourhood $W \subset B \times D$ of $0 \in \mathbb{C}^{m-n}$ and a constant $A>0$ such that

$$
(x, y) \in \pi(Z) \cap W \Rightarrow|y| \leq A|x| .
$$

After these preparations let us define

$$
Q=\left\{q>0: \exists \widetilde{c}>0:|z|+|y| \leq \widetilde{c}|x|^{q} \text { for }(x, y, z) \in Z\right.
$$

in some neighbourhood of 0$\}$.

By ([7], Th. (1.2)) we get:

$\left.1^{\prime}\right) q_{0}=\sup Q \in(Q \cap \mathbb{Q}) \cup\{+\infty\}$, 
$\left.2^{\prime}\right) d^{-1} \in Q$

where $d=i(Z \cdot N ; 0)-\operatorname{deg}_{0} Z+1$.

Now, observe that Lemma 1.2 implies that $Z$ and $N$ are $p$-separated at $0 \in \mathbb{C}^{m}$ if there exists $c>0$ such that

$$
|x|+|y| \geq c(|x|+|y|+|z|)^{p} \quad \text { for }(x, y, z) \in Z
$$

in some neighbourhood of $0 \in \mathbb{C}^{m}$.

We prove that

$$
P=\{1 / q: q \in Q, q \leq 1\} .
$$

First, suppose that $q \in Q, q \leq 1$. Then $p=1 / q \geq 1$ and $|x| \geq c_{1}(|z|+|y|)^{p}$ for $(x, y, z) \in Z$ in some neighbourhood of 0 and for some constant $c_{1} \in(0,1)$. This implies $|x| \geq\left(c_{1} / 2^{p}\right)(|x|+|y|+|z|)^{p}$ and finally, there exists $c_{2}>0$ such that $|x|+|y| \geq c_{2}(|x|+|y|+|z|)^{p}$ for $(x, y, z) \in Z$ in some neighbourhood of 0 . Hence $p=1 / q \in P$.

Now, let $p \in P$. Then $p \geq 1$ and there exists $c>0$ such that

$$
|x|+|y| \geq c(|x|+|y|+|z|)^{p} \quad \text { for }(x, y, z) \in Z
$$

in some neighbourhood of 0 . By property $(*)$ we get

$$
|x| \geq c_{3}(|y|+|z|)^{p},
$$

and finally there exists $c_{4}>0$ such that

$$
|y|+|z| \leq c_{4}|x|^{q}, \quad \text { where } q=1 / p,
$$

for $(x, y, z) \in Z$ in some neighbourhood of 0 . Therefore $p=1 / q$ where $q \in Q$ and $q \leq 1$, which proves $(* *)$. Since $d \geq 1$, condition $\left.2^{\prime}\right)$ implies $d \in P$.

It is easily seen that $p_{0}=\max \left\{1,1 / q_{0}\right\} \leq d$. From $1^{\prime}$ ) we conclude that $p_{0} \in P \cap \mathbb{Q}$, and the proof is complete.

In the remainder of this paper we assume that $X$ and $Y$ are analytic subsets of an $m$-dimensional $(m \geq 1)$ complex manifold $M$, and that $a$ is an isolated point of $X \cap Y$.

Define

$$
P=\{p>0: X \text { and } Y \text { are } p \text {-separated at } a\} .
$$

We can now state our main result.

Theorem 3.2. If $X$ and $Y$ are pure dimensional, then

1) $p_{0}=\inf P \in P \cap \mathbb{Q}$,

2) $1 \leq p_{0} \leq \mathfrak{i}(X \cdot Y ; a)-\operatorname{deg}_{a} X \cdot \operatorname{deg}_{a} Y+1$.

Pro of. Define

$$
\begin{gathered}
Z=X \times Y \subset M^{2}, \quad N=\Delta_{M} \subset M^{2}, \\
\widetilde{P}=\{p>0: Z \text { and } N \text { are } p \text {-separated at }(a, a)\} .
\end{gathered}
$$


By Lemma 1.3, $P=\widetilde{P}$. It is obvious that $i(X \cdot Y ; a)=\mathfrak{i}\left(Z \cdot \Delta_{M} ;(a, a)\right)$ and $\operatorname{deg}_{(a, a)} Z=\operatorname{deg}_{a} X \cdot \operatorname{deg}_{a} Y$. Now, Theorem 3.1 completes the proof.

In the last two theorems we have been working under the assumption that $X, Y$ are pure dimensional. To study the general case suppose that $X_{1}, \ldots, X_{r}$ and $Y_{1}, \ldots, Y_{s}$ are all components of $X$ and $Y$, respectively, passing through $a$. We can extend our definitions from the pure dimensional case (cf. [1]) by the following natural formulas:

$$
\begin{gathered}
i(X \cdot Y ; a)=\sum_{k=1}^{r} \sum_{l=1}^{s} i\left(X_{k} \cdot Y_{l} ; a\right), \\
\operatorname{deg}_{a} X=\sum_{k=1}^{r} \operatorname{deg}_{a} X_{k}, \quad \operatorname{deg}_{a} Y=\sum_{l=1}^{s} \operatorname{deg}_{a} Y_{l} .
\end{gathered}
$$

We can now state the analogue of the last theorem.

Corollary 3.3. Under the above definitions:

1) $p_{0}=\inf P \in P \cap \mathbb{Q}$,

2) $1 \leq p_{0} \leq \mathfrak{i}(X \cdot Y ; a)-\operatorname{deg}_{a} X \cdot \operatorname{deg}_{a} Y+1$.

Proof. It is clear that $p_{0}=\max \left\{p_{0}\left(X_{k}, Y_{l} ; a\right): k=1, \ldots, r, l=\right.$ $1, \ldots, s\}$ (see Section 1), which implies 1), by Theorem 3.2. Let $p_{0}=$ $p_{0}\left(X_{k}, Y_{l} ; a\right)$ for some fixed $k, l$. Observe that Theorem 3.2 gives

$$
1 \leq p_{0}=p_{0}\left(X_{k}, Y_{l} ; a\right) \leq i\left(X_{k} \cdot Y_{l} ; a\right)-\operatorname{deg}_{a} X_{k} \cdot \operatorname{deg}_{a} Y_{l}+1 .
$$

An easy computation shows that

$$
i\left(X_{k} \cdot Y_{l} ; a\right)-\operatorname{deg}_{a} X_{k} \cdot \operatorname{deg} Y_{l} \leq i(X \cdot Y ; a)-\operatorname{deg}_{a} X \cdot \operatorname{deg}_{a} Y,
$$

and the proof is complete.

The following corollary yields information about "1-separation" in terms of tangent cones of sets.

COROLlary 3.4. The following conditions are equivalent:

1) $X$ and $Y$ are 1-separated at a,

2) $C_{a}(X) \cap C_{a}(Y)=\{0\}$.

Proof. Without loss of generality we can assume that $M$ is an open subset of $\mathbb{C}^{m}$ and that $a=0$.

First, suppose that $X$ and $Y$ are 1-separated at 0 and, by contradiction, that $v \in C_{0}(X) \cap C_{0}(Y), v \neq 0$. This implies $(v, v) \in C_{0}(X \times Y) \cap \Delta_{\mathbb{C}^{m}}$ and so, by definition, there exist sequences $\left(x_{\nu}, y_{\nu}\right) \in X \times Y$ and $\lambda_{\nu} \in \mathbb{C}$ such that

$$
x_{\nu} \rightarrow 0, y_{\nu} \rightarrow 0, \lambda_{\nu}\left(x_{\nu}, y_{\nu}\right) \rightarrow(v, v) \quad \text { as } \nu \rightarrow \infty .
$$


Since $X$ and $Y$ are 1-separated, $\left|x_{\nu}-y_{\nu}\right| \geq C\left|x_{\nu}\right|$ for some $c>0$ and sufficiently large $\nu$. Then $\left|\lambda_{\nu} x_{\nu}-\lambda_{\nu} y_{\nu}\right| \geq C\left|\lambda x_{\nu}\right|$, which is impossible.

Next, if $C_{0}(X) \cap C_{0}(Y)=\{0\}$ then ([1], Th. 5.6) implies $\mathfrak{i}(X \cdot Y ; 0)=$ $\operatorname{deg}_{0} X \cdot \operatorname{deg}_{0} Y$. By Corollary 3.3 we get $p_{0}(X, Y ; 0)=1$, which completes the proof.

We shall now construct an example showing that the estimate of $p_{0}$ presented in our basic Theorem 3.1 is optimal.

Example 3.5. Let $s \geq d \geq 1$ be integers. Define $M=\mathbb{C}^{2}, a=0$ and $Z=\left\{(x, y) \in \mathbb{C}^{2}: y^{s}+x y^{d-1}+x^{d}=0\right\}, \quad N=\left\{(x, y) \in \mathbb{C}^{2}: x=0\right\}$.

Straightforward calculation yields that $\operatorname{deg}_{0} Z=d, \mathfrak{i}(Z \cdot N ; 0)=s$ and

$$
p_{0}=p_{0}(Z, N ; 0)=s-d+1 \text {. }
$$

\section{References}

[1] R. Achilles, P. Tworzewski and T. Winiarski, On improper isolated intersection in complex analytic geometry, Ann. Polon. Math. 51 (1990), 21-36.

[2] R. Draper, Intersection theory in analytic geometry, Math. Ann. 180 (1969), 175204 .

[3] S. Łojasiewicz, Ensembles semi-analytiques, I.H.E.S., Bures-sur-Yvette, 1965.

[4] -, Introduction to Complex Analytic Geometry, Birkhäuser, Basel 1991.

[5] —, Sur la séparation régulière, Univ. Studi Bologna, Sem. Geom. 1985, 119-121.

[6] A. Płoski, Multiplicity and the Lojasiewicz exponent, preprint 359, Polish Academy of Sciences, Warszawa 1986.

[7] -, Une évaluation pour les sous-ensembles analytiques complexes, Bull. Polish Acad. Sci. Math. 31 (1983), 259-262.

[8] P. Tworzewski and T. Winiarski, Analytic sets with proper projections, J. Reine Angew. Math. 337 (1982), 68-76.

[9] T. Winiarski, Continuity of total number of intersection, Ann. Polon. Math. 47 (1986), 155-178.

INSTITUTE OF MATHEMATICS

JAGIELLONIAN UNIVERSITY

REYMONTA 4

30-059 KRAKÓW, POLAND 